for the metal-semiconductor transition, which involves a localized state and a conduction-band state whose energy separation decreases with temperature or pressure. They define an effective energy gap given by the equation $\Delta_{\text {eff }}=\Delta$ $-2 G n$, where $\Delta$ is the energy gap at $T=0$ and $P=0, G$ is the strength of electron-hole interaction, and $n$ is the number density of electrons excited into the conduction band. According to this model the number $n$ of electrons in the conduction band can vary continuously or discontinuously with temperature at $P=0$, depending on the relative values of $G$ and $\Delta$. Bucher ${ }^{10}$ has recently extended these ideas and has shown that the model can lead to first- and second-order electronic phase transitions at $T=0$ when pressure is varied. The parameter $G$, which is a measure of the strength of electron-hole interaction, has to exceed a certain critical value compared to the total band energy, in order to get a firstorder transition under pressure. YbTe has a gap of about $2 \mathrm{eV}$, and apparently in materials with large gap the ratio $G / \Delta$ is below the critical value for a discontinuous transition.

Europium telluride also has a gap of about $2 \mathrm{eV}$, and Rooymans ${ }^{11,12}$ reported a discontinuous electronic phase transition involving the conversion of an $\mathrm{Eu}$ ion to the trivalent state near $30 \mathrm{kbar}$. However, our $p-V$ data $^{4}$ on EuTe up to $100 \mathrm{kbar}$ do not show any evidence of an electronic transition. Near $100 \mathrm{kbar}$ the NaCl-type structure transforms to the $\mathrm{CsCl}$ structure. ${ }^{4}$ Hence, it ap- pears from the behavior of YbTe that if the energy gap is large, a continuous electronic transition would be favored in the rare-earth chalcogenides.

One of us (A.J.) would like to thank the John Simon Guggenheim Foundation for a fellowship. We would like to thank Miss Usha Devi for assistance in $\mathrm{x}$-ray data processing.

\footnotetext{
${ }^{1}$ A. Jayaraman, E. Bucher, and D. B. McWhan, in Proceedings of the Eighth Rave Earth Research Conference, edited by T. A. Henrie and R. E. Lindstrom (U. S. GPO, Washington, D. C., 1970), Vol. 1, p. 333.

${ }^{2}$ A. Jayaraman, V. Narayanamurti, E. Bucher, and R. G. Maines, Phys. Rev. Lett. 25, 368 (1970).

${ }^{3}$ A. Jayaraman, V. Narayanamurti, E. Bucher, and R. G. Maines, Phys. Rev. Lett. 25, 1430 (1970).

${ }^{4}$ A. K. Singh, A. Jayaraman, and A. Chatterjee, to be published.

${ }^{5}$ W. A. Bassett, T. Takahashi, and P. W. Stook, Rev. Sci. Instrum. 38, 37 (1967).

${ }^{6}$ M. Francillon, D. Jerome, J. C. Achard, and G. Malfait, J. Phys. (Paris) 31, 709 (1970).

${ }^{7}$ R. Suryanarayanan and C. Paparoditis, Solid State Commun. 8, 1853 (1970).

${ }^{8}$ C. C. Wong and C. Wood, Phys. Lett. 34A, 125 (1971).

${ }^{9}$ L. M. Falicov and J. C. Kimball, Phys. Rev. Lett. 22, 997 (1969).

${ }^{10} \mathrm{E}$. Bucher, to be published.

${ }^{11}$ C. J. M. Rooymans, Solid State Commun. 3,421 (1965).

${ }^{12}$ C. J. M. Rooymans, Ber. Bunsenges. Phys. Chem. 70, 1036 (1966).
}

\title{
Properties of the Interstitial in the Diamond-Type Lattice
}

\author{
G. D. Watkins and R。P. Messmer \\ General Electric Corporate Research and Development, Schenectady, New York 12301 \\ and \\ C. Weigel, D. Peak, and J. W. Corbett* \\ Department of Physics, State University of New York at Albany, Albany, New York 12203
}

(Received 27 October 1971)

\begin{abstract}
Linear combination of atomic orbital-molecular orbital cluster calculations using extended Hïckel theory suggest that the interstitial carbon atom in diamond prefers an "interstitialcy" configuration. The predicted minimum-energy configuration changes with charge state, providing a possible example of the Bourgoin mechanism for "athermal" migration of the interstitial in the presence of ionizing radiation.
\end{abstract}

Considerable interest and mystery surround the properties of an interstitial host atom in the diamond-type lattice. This fundamental defect must be formed, for instance, as the Frenkel partner to the lattice vacancy in a radiation-damage experiment. In silicon, lattice vacancies produced in such experiments have been detected directly by EPR. ${ }^{1-3}$ However, the interstitials 
have so far eluded detection. In fact, no direct experimental information on the isolated hostatom interstitial is available for any material of the diamond lattice.

The reason for this is not understood and is of considerable current concern. One suggestion ${ }^{2}$ put forth for silicon is that the interstitial is mobile even at $4.2^{\circ} \mathrm{K}$ and therefore does not remain in the lattice during a radiation-damage experiment. The evidence for this is the observation that interstitial group-III atoms can be produced in a $4.2^{\circ} \mathrm{K}$ irradiation. ${ }^{2}$ In this model, the silicon interstitial formed in the damage event migrates until it encounters a substitutional group-III atom, whereupon it trades places, ejecting the impurity into the interstitial site. Models involving low-temperature, long-range migration of interstitials in germanium have also been considered. ${ }^{4}$ The mechanism for such a low-temperature migration remains a mystery, however.

An alternative explanation is that interstitials are present but are electrically inactive. This possibility has been revived recently by Singhal ${ }^{5}$ who, using an extended Koster-Slater approach, ${ }^{6}$ solved for the electronic structure of a silicon atom in the tetrahedral interstitial position and found no localized state in the gap.

In this Letter we describe preliminary results of calculations performed on the interstitial carbon atom in diamond, which we feel may provide new insight into this problem. In these calculations we have used a linear combination of atomic orbital-molecular orbital (LCAO-MO) cluster approach which has been previously described and shown to be successful for treating the electronic structure of the deep levels produced by substitutional impurities, ${ }^{7,8}$ and the lattice vacancy in diamond. ${ }^{9}$ As in the previous studies, we have used extended Hückel theory ${ }^{10}$ (EHT) with clusters of up to 35 carbon atoms centered around the defect. In investigating the stability of various defect configurations, we monitor the total EHT energy as given by the sum of the occupied one-electron molecular orbitals. ${ }^{11}$

We describe here the results for the four different interstitial configurations illustrated in Fig. 1. For the "interstitialcy" configurations $c$ and $d$, the atoms directly involved (shown shaded) were allowed to relax symmetrically for minimum energy. All other atoms were held fixed in their regular lattice positions.

The results for the neutral charge state $\mathrm{C}^{0}$ are given in Table I. We note that the two interstitialcy sites are predicted to be very much lower

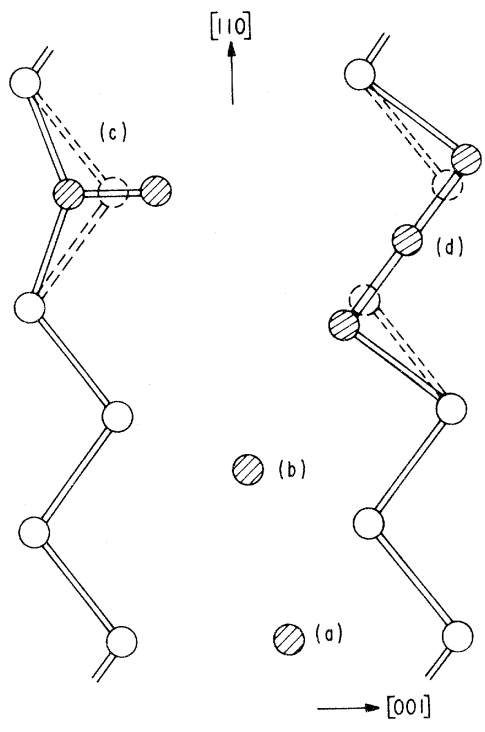

FIG. 1. The [110] plane in diamond showing the four interstitial configurations considered: $a$, an atom in the tetrahedral site, $b$, an atom in the hexagonal site, $c$, a "split- $\langle 100\rangle$ " configuration, and $d$, a "bond-centered" configuration.

in energy $(\sim 10 \mathrm{eV})$ than the hexagonal site which in turn is correspondingly lower than the tetrahedral site. It is difficult to estimate the accuracy of calculations such as these where an approximate scheme such as EHT is used. However, this is very similar to the type of calculation for which EHT has proven to be remarkably reliable-that of predicting conformational stability in molecules ${ }^{10}$-and we suggest therefore that such large energy differences should be taken seriously.

We interpret these results to indicate that "interstitialcy" configurations are preferred. This is an important result because all previous theoretical treatments of the interstitial have assumed the tetrahedral $1^{5,12-17}$ or hexagonal $1^{15,16}$ sites. Speculations as regards interstitialcy configura-

TABLE I. Relative EHT total energies (eV) for four different interstitial configurations (see Fig. 1). The numbers in parentheses are a guide to the properties of the states $\mathrm{C}^{+}$and $\mathrm{C}^{-}$(see text).

\begin{tabular}{ccccc}
\hline & & & $c$ & \\
State & $\begin{array}{c}c \\
\text { Tetrahedral }\end{array}$ & $\begin{array}{c}d \\
\text { Hexagonal }\end{array}$ & $\begin{array}{c}\text { Split } \\
\langle 100\rangle\end{array}$ & $\begin{array}{c}\text { Bond } \\
\text { centered }\end{array}$ \\
\hline $\mathrm{C}^{0}$ & 0 & -9.1 & -21.7 & -21.7 \\
$\mathrm{C}^{+}$ & 0 & $(-10.8)$ & $(-19.4)$ & $(-20.9)$ \\
$\mathrm{C}^{-}$ & 0 & $(-7.4)$ & $(-24.2)$ & $(-22.8)$ \\
\hline
\end{tabular}


tions have been given previously in the literature ${ }^{18-20}$ but we believe our results represent the first estimates of their stability.

We note also that the predicted stability of the two neutral interstitialcy sites is identical. Although this exact agreement must be fortuitious, it clearly points to the possibility of easy motion through the lattice since the transition from one to the other represents the logical path for interstitialcy migration. Interstitialcy migration also fits in well with the group-III atom-ejection model in that the extra atom is already in a bonded configuration, making a final activation barrier less likely.

Our results also predict localized electronic states in the forbidden gap. The levels are well removed from the band edges of the cluster and in the neutral charge state are partially filled. This suggests that the defect may act as both donor and/or acceptor. EHT is not a self-consistent treatment and as such it approximates best the neutral charge state of the defect $\left(C^{0}\right)$, where the charge density is uniformly distributed over the cluster. However, a guide to the properties of the ionized states $\mathrm{C}^{+}$and $\mathrm{C}^{-}$can be obtained by removing or adding, respectively, an electronic charge to the cluster.

The results, also given in the table, predict that the relative stability of the two interstitialcy sites switches substantially $(\sim 1.5 \mathrm{eV})$ from one to the other depending upon whether the defect is negatively or positively charged. This intriguing result argues that the Bourgoin mechanism ${ }^{21}$ for low-temperature migration of the interstitial may apply to these interstitialcy configurations. That is, in a high-energy charged-particle irradiation an immense amount of ionization is produced in addition to simple lattice displacements. The alternate capture of electrons and holes by the interstitial can therefore serve to drive it from one configuration to the other, supplying an athermal mechanism for its motion.

A full account of these results and also those for interstitial boron and nitrogen in diamond is in preparation and will be published elsewhere. ${ }^{22}$

*Work partially supported by the U. S. Office of Naval Research under Contract No. (N00014-70C-0296).
${ }^{1}$ G. D. Watkins, J. Phys. Soc. Jap. 18, Suppl. II, 22 (1963).

${ }^{2}$ G. D. Watkins, in Radiation Damage in Semiconductors (Dunod, Paris, 1965), p. 97.

${ }^{3}$ G. D. Watkins, in Radiation Effects in Semiconductors, edited by F. L. Vook (Plenum, New York, 1968), p. 67 .

${ }^{4}$ See the recent review by J. W. MacKay and E. E. Klontz, Radiat. Eff. $\underline{9}, 27$ (1971).

${ }^{5}$ S. P. Singhal, Phys. Rev. B, 4 , 2497 (1971).

${ }^{6} \mathrm{~J}$. Callaway and A. J. Hughes, Phys. Rev. 156, 860 (1967).

${ }^{7}$ R. P. Messmer and G. D. Watkins, Phys. Rev. Lett. $\underline{25}, 656$ (1970) .

${ }^{8} \mathrm{G}$. D. Watkins and R. P. Messmer, in Proceedings of the Tenth International Conference on the Physics of Semiconductors, Cambridge, Massachusetts, 1970, edited by S. P. Keller, J. C. Hensel, and F. Stern, CONF-700801 (U. S. AEC Division of Technical Information, Springfield, Va., 1970), p. 623.

${ }^{9}$ R. P. Messmer and G. D. Watkins, Radiat. Eff. $\underline{9}, 9$ (1971).

${ }^{10}$ R. Hoffmann, J. Chem. Phys. 39, 1397 (1963).

${ }^{11}$ The use of EHT in this manner to deduce such physical properties as the stability, migration, and formation energies of defects in solids was first demonstrated by E. B. Moore and C. M. Carlson [Solid State Commun. $\underline{4}, 47$ (1965)] for defects in the graphite lattice.

${ }^{12}$ H. M. James and K. Lark-Horovitz, Z. Phys. Chem. 198, 107 (1951).

${ }^{13}$ E. I. Blount, Phys. Rev. 113,995 (1959).

${ }^{14} \mathrm{~T}$. Yamaguchi, J. Phys. Soc. Jap. 18, 386, 923

(1963).

${ }^{15}$ K. H. Bennemann, Phys. Rev. 137, A1497 (1965), and 139 , A482 (1965).

${ }^{16}$ R. R. Hasiguti, J. Phys. Soc. Jap. 21, 1927 (1966).

${ }^{17}$ K. Schroeder, Bull. Amer. Phys. Soc. 16, 397 (1971).

${ }^{18} \mathrm{G}$. D. Watkins, as described by J. W. Corbett, Electron Radiation Damage in Semiconductors and Metals (Academic, New York, 1966), p. 99.

${ }^{19} \mathrm{~J}$. Friedel, in Radiation Effects on Semiconductor Components (Journées d'Electronique, Toulouse, France, 1967), Vol. I, p. A11.

${ }^{20} \mathrm{~J}$. W. Corbett, in Radiation Effects in Semiconductors, edited by F. L. Vook (Plenum, New York, 1968), p. 3 .

${ }^{21} \mathrm{~J}$. Bourgoin, as reported by J. W. Corbett, in Proceedings of the Annual Conference on Nuclear and Space Radiation Effects, Durham, New Hampshire, July 1971 (unpublished); J. Bourgoin and J. W. Corbett, to be published. Bourgoin considered charge-state conversion between the tetrahedral and hexagonal sites.

${ }^{22}$ C. Weigel, D. Peak, J. W. Corbett, R. P. Messmer, and G. D. Watkins, to be published. 